\title{
Acknowledgment to Reviewers of Sports in 2021
}

\author{
Sports Editorial Office
}

Citation: Sports Editorial Office. Acknowledgment to Reviewers of Sports in 2021. Sports 2022, 10, 20. https://doi.org/10.3390/

sports 10020020

Published: 31 January 2022

Publisher's Note: MDPI stays neutral with regard to jurisdictional claims in published maps and institutional affiliations.

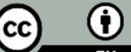

Copyright: $\odot 2022$ by the author. Licensee MDPI, Basel, Switzerland. This article is an open access article distributed under the terms and conditions of the Creative Commons Attribution (CC BY) license (https://creativecommons.org/licenses/by/4.0/).

MDPI AG, St. Alban-Anlage 66, 4052 Basel, Switzerland

Rigorous peer-reviews are the basis of high-quality academic publishing. Thanks to the great efforts of our reviewers, Sports was able to maintain its standards for the high quality of its published papers. Thanks to the contribution of our reviewers, in 2021, the median time to first decision was 22 days and the median time to publication was 46 days. The editors would like to extend their gratitude and recognition to the following reviewers for their precious time and dedication, regardless of whether the papers they reviewed were finally published:

Aaron Scanlan

Abdul Rashid Aziz

Adam Gorman

Adam White

Adela Badau

Adrian A. Valverde

Agnieszka Zembrón-Łacny

Aïmen Khacharem

Ajay Palagani

Alban Fouasson-Chailloux

Alejandro Martínez-Rodriguez

Alejandro Pérez-Castilla

Alejandro Sánchez-Pay

Aleksandra Królikowska

Aleksandra Rogowska

Alessandro Feola

Alex Buoite Stella

Alfonso Penichet-Tomás

Ali Boolani

Alina Kuryłowicz

Alvaro Lopez-Samanes

Anastassios Philippou

Andras Papp

André Novo

Andrea Fusco

Andrea Vitali

Andrew D'Lugos

Andrew Hatchett

Andrew Hulton

Andrew J. Harrison

Andrew Lavender

Andrzej Mastalerz

Anna Leopold

Anna Tabęcka-Łonczyńska
Anthony Hessel

Antonio Cicchella

António Mendo

Argyris Toubekis

Arkadiusz Stanula

Arkaitz Castañeda

Arne Güllich

Arthur Chaves

Ashley Artese

Asrat Amnie

Athos Trecroci

Badicu Georgian

Basilio Pueo

Blanca De-la-Cruz-Torres

Brach Poston

Brandon J. Sawyer

Britton W. Brewer

Bruce W. Craig

Bruno Travassos

Bryanne N. Bellovary

Bud Cooper

Caio Victor Sousa

Carl Singleton

Carlos David Gómez-Carmona

Carlos Eduardo Barro Gonçalves

Carlos López-de-Celis

Carlos Romero Morales

Carole Cometti

Cesar I. Fernández Lázaro

Che Fornusek

Chia-Hua Kuo

Ching-Ting Hsu

Chong Chen

Christian Roques 
Christoph Triska

Christopher Ballmann

Christopher Kirk

Christos Paizis

Claudia Leong

Clayton L. Camic

Cornelis Johannes De Ruiter

Courtney E. Shell

Craig A. Horswill

Cristina Cortis

Cristina Petisco-Rodríguez

Dagmar Pavlu

Daichi Sumi

Daichi Yamashita

Dale Chapman

Damien Young

Daniel A. Barone

Daniel Becque

Daniel Hackett

Daniel López-López

Dariusz Boguszewski

Dariusz Nowak

David Behm

David Berry

David H. Fukuda

David Niederseer

David Zweiker

Deldicque Louise

Dennis Peter Born

Diego Alonso-Fernandez

Diego Fernández-Lázaro

Dolores D. Guest

Donatella Di Corrado

Dorota Kostrzewa-Nowak

Dorota Lewczuk

Duarte Henriques-Neto

Dustin Russel Slivka

Eleftherios Kellis

Elena Escolano-Pérez

Emanuela Gualdi-Russo

Emiliano Cè

Emmanuel Navarro-Flores

Enrique Roche

Enza Speranza

Eric Berkson

Eric Mosier

Eric Sobolewski

Erica Menegatti

Erik Lind

Erika Zemková

Euphrosyni Koutsouraki
Fabiola Spolaor

Felice Sirico

Fernando González-Mohíno

Filipe Manuel Clemente

Fotini Arabatzi

Francesca Latino

Francesco Agostini

Francesco Fischetti

François Lalonde

Frank E. Nelson

Frank Wyatt

František Lopot

Fraser Carson

Gaetana Napolitano

Garry Kuan

Gary Brickley

Gary Liguori

Gennady Kolesnikov

Geoffrey Istas

Gerard McMahon

Gerd Schmitz

Giampaolo Santi

Giancarlo Condello

Gianluca Esposito

Gianpiero Greco

Giovanna Ghiani

Giuseppe Caminiti

Giuseppe Coratella

Goran Kuvačić

Govindasamy Balasekaran

Gregory Bogdanis

Gregory J. Grosicki

Gustavo Vieira De Oliveira

Gusztáv Fekete

Haneul Lee

Helder Miguel Fernandes

Henk Koppelaar

Henri Tilga

Henrique Nascimento

Henry Lukaski

Hermann Toplak

Hugo Sarmento

Ibrahim Hassan

Ignacio Refoyo

Igor Jukić

Ioana Mozos

Irene Margaritis

Irene Torres-Sánchez

Ismael Guardado

Iván Chulvi-Medrano

Jacek Jurkojć 


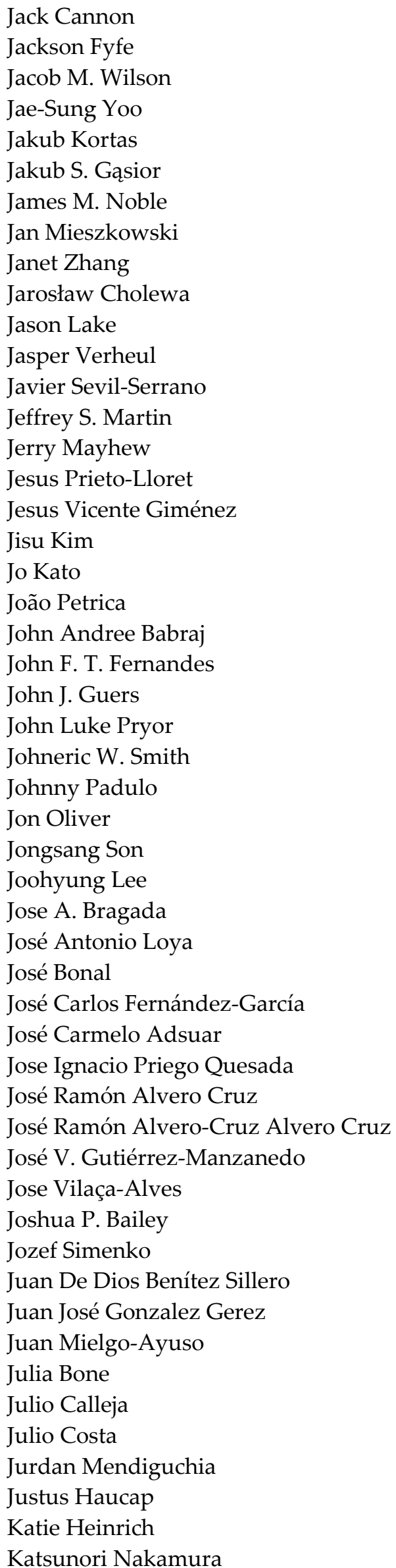

Kazuhiro P. Izawa

Kazuki Fukui

Kevin Till

Khaled Trabelsi

Konstantinos Papanikolaou

Kristina Penniston

Krzysztof Jamroziak

Krzysztof Kusy

Krzysztof Sas-Nowosielski

Kuen-Chang Hsieh

Lachlan Mitchell

Larisa-Loredana Dragolea

Laurent Metzinger

Lawrence Judge

Leonor Gallardo

Liliana Szyszka-Sommerfeld

Liudmila I. Gerasimova-Meigal

Luca Ardigò

Luca Cavaggioni

Luca Paolo Ardigò

Luciana Labanca

Luigi Meccariello

Luis Carrasco

Luis Javier Chirosa Ríos

Luís Rama

Łukasz Radzimiński

Magdalena Górnicka

Małgorzata Bronikowska

Malgorzata Syczewska

Manuel Albornoz-Cabello

Marc Argilés

Marc Lochbaum

Marco Gervasi

Marco J.M. Hoozemans

Marcos Mecías-Calvo

Margaret Jones

Maria Francesca Piacentini

María Luisa Zagalaz Sánchez

María M. Morales Suárez-Varela

Marieke Wouters

Mário Jorge Costa

Mark Elisabeth Theodorus Willems

Mark Russell

Marko Stupin

Martin Otis

Masatoshi Nakamura

Mathieu Jozwiak

Mati Pääsuke

Matt R. Cross

Matteo Levi Micheli

Matthew Cook 
Matthew H. Zimmerman

Matthew J. Reeves

Matthew Rogatzki

Meike Streker

Micah Gross

Michael J. Duncan

Michael Keiner

Michael Kingsley

Michael Olson

Michaela Šiklová

Michał Bronikowski

Michał Krzysztofik

Michal Starczewski

Michale Koehle

Michalina Błażkiewicz

Michel Marina

Michele Boffano

Miguel Angel Gómez-Ruano

Mike Climstein

Mikel Pérez Gutiérrez

Mikolaj Dabrowski

Milivoj J. Dopsaj

Mitchell Lachlan

Mojtaba Kaviani

Motomu Nakashima

Nai-Jen Chang

Natasa Zenic

Navarro Gómez Noelia

Nicholas Gist

Nicolas Babault

Nikolai Baastrup Nordsborg

Ozcan Esen

Pablo Burillo

Pål Lagestad

Panagiotis Tsimeas

Pantelis T. Nikolaidis

Paolo Riccardo Brustio

Pasqualino Maietta Latessa

Paul Dorian

Paulo Vicente João

Paweł Chmura

Pawel Macek

Pedro Fonseca

Pedro L. Valenzuela

Pedro Sáenz-Lopez

Pedro Valdivia-Moral

Pei-Lun Kuo

Peter Hofmann

Peter Sarcevic

Petr Stastny

Petros Botonis
Phil Chilibeck

Phil J. Handcock

Pierpaolo Iodice

Pietro Picerno

Piotr Gawda

Pitre C. Bourdon

Piyush Sharma

Prasenjit Saha

Rafael Burgueño

Rafael Oliveira

Ramos-Petersen Laura

Raquel Leirós-Rodríguez

Raul Domínguez

Raúl Tárraga Mínguez

Rebecca Williams

Ren-Jay Shei

Ricardo Manuel Pires Ferraz

Robert Alan Sloan

Robert F. Bentley

Roland Van Den Tillaar

Román Nuviala

Romualdas Malinauskas

Rosario Barone

Russell Best

Ryan Colquhoun

Ryan M. Sapp

Ryan Worn

Ryu Nagahara

Sabrina Donati Zeppa

Sai-fu Fung

Salvador Romero-Arenas

Samo Rauter

Samuel Mettler

Scot Raab

Scott C. Forbes

Scott Mazzetti

Scott W. Talpey

Sébastien Murer

Sergio López-García

Sergio Selles-Perez

Seungyong Lee

Shaher A. I. Shalfawi

Shalaka Mulherkar

Shih-yu Lee

Shinsuke Nirengi

Shin-Tsu Chang

Sihui Ma

Silvio Lorenzetti

Sime Versic

Soo Jin Yang

Stefano Amatori 
Stephan Van Der Zwaard

Stéphane Perrey

Stephen Cornish

Stephen Ives

Steve Thompson

Szabo Attila

Tadashi Ito

Tadeusz Ambrozy

Tarak Driss

Tatiana Görig

Tatsuro Egawa

Teresa Zwierko

Thanutchaporn Kumrungsee

Themistoklis Tsatalas

Thomas Bias

Thórarinn Sveinsson

Tim Marjoribanks

Timothy B. Davies

Timothy J. Suchomel
Tobias Dünnwald

Tomás Freitas

Tomás Urbán

Tomasz Gabryś

Tomasz Klekiel

Tomasz Wolny

Toshinobu Takeda

Toshio Moritani

Tsering Stobdan

Vaclav Bunc

Weisheng Chiu

Wesley Kephart

Wojciech J. Cynarski

Xiao Chang

Xiaohu Huang

Xurxo Dopico

Yong-Seok Jee

Yoshinari Ueha 